\title{
Caregiver burden in late-stage parkinsonism and its associations
}

Stefania Kalampokini, MD, MSc, PhD; Adrianus LAJ. Hommel, MSc²; Stefan Lorenzl, MD³ Joaquim J. Ferreira, MD, PhD ; Wassilios G. Meissner, MD, PhD ${ }^{5,6}$, Per Odin MD, PhD ${ }^{7}$, Bastiaan R. Bloem, MD, $\mathrm{PhD}^{2}$, Richard Dodel $\mathrm{MD}^{8}$, Anette-Eleonore Schrag FRCP, $\mathrm{PhD}^{1 *}$ and the CLaSP consortium

${ }^{1}$ UCL Queen Square Institute of Neurology, University College London, Rowland Hill street, NW3 2PF, London, UK.

2Department of Neurology, Donders Institute for Brain, Cognition and Behavior, Radboud University Nijmegen Medical Centre, Nijmegen, The Netherlands.

${ }^{3}$ Institute of Nursing Science and Practice, Paracelsus Medical University, Salzburg, Austria. Interdisziplinäres Zentrum für Palliativmedizin und Klinik für Neurologie Universität München- Klinikum Großhadern, Munich, Germany. Department of Neurology, Agatharied Hospital, Hausham, Germany

${ }^{4}$ Instituto de Medicina Molecular Universidade di Lisboa, Lisboa, Portugal.

${ }^{5}$ Service de Neurologie, CHU de Bordeaux, 33000, Bordeaux, France. Institut des Maladies Neurodégénératives, University de Bordeaux, UMR 5293, 33000, Bordeaux, France.

${ }^{6}$ Dept. Medicine, University of Otago, Christchurch, New Zealand and New Zealand Brain Research Institute, Christchurch, New Zealand

${ }^{7}$ Department of Neurology, Lund University Hospital, Lund, Sweden.

${ }^{8}$ Department of Geriatric Medicine, University Duisburg-Essen and Department of Neurology, Philipps-University Marburg, Germany.

*corresponding author

The Care of Late Stage Parkinsonism (CLaSP) study is a longitudinal, multicenter, prospective cohort study conducted in six European countries (United Kingdom, France, Germany, Netherlands, Portugal, Sweden). The study was funded by the European Commission (Joint Programme- Neurodegenerative Disease Research "European research projects for the evaluation of health care policies, strategies and interventions for Neurodegenerative Diseases") through national funding bodies in all six countries (Economic and Social Research Council ES/L009250/1; BMBF, Marburg, Germany 01ED1403A, Munich, Germany 01ED1403B, Bordeaux, France: ANR-13-JPHC-0001-07, Lisbon, Portugal: HC/ 0002/2012, Lund, Sweden: HC-559-002, Nijmegen, Holland, 
733051003). AS was supported by the National Institute for Health Research UCL/UCLH Biomedical Research Centre. AH was supported by co-funding of Groenhuysen organization and Stichting Beroepsopleiding Huisartsen.

\section{Abstract}

Background: Patients in the late stages of parkinsonism are highly dependent on others in their self-care and activities of daily living. However, few studies have assessed the physical, psychological and social consequences of caring for a person with late-stage parkinsonism.

Patients and methods: Five hundred and six patients and their caregivers from the Care of Late Stage Parkinsonism (CLaSP) study were included. Patients' motor and non-motor symptoms were assessed using the UPDRS and Non-motor symptom scale (NMSS), Neuropsychiatric inventory (NPI-12), and caregivers' health status using the EQ-5D-3L. Caregiver burden was assessed by the Zarit Burden Interview (ZBI).

Results: The majority of caregivers were the spouse or life partner (71.2\%), and were living with the patient at home (67\%). Approximately half of caregivers reported anxiety/depression and pain/discomfort ( $45 \%$ and $59 \%$ respectively). The factors most strongly associated with caregiver burden were patients' neuropsychiatric features on the total NPI score $(r=0.38$, $p<0.0001)$, total NMSS score $(r=0.28, p<0.0001)$, caring for male patients and patients living at home. Being the spouse, the hours per day assisting and supervising the patient as well as caregivers' EQ-5D mood and pain scores were also associated with higher ZBI scores (all $p<0.001)$

Conclusion: The care of patients with late stage parkinsonism is associated with significant caregiver burden, particularly when patients manifest many neuropsychiatric and non-motor features and when caring for a male patient at home. 
Keywords: late-stage parkinsonism, caregiver burden, neuropsychiatric, non-motor symptoms

\section{Introduction}

Parkinson's disease (PD) is a common neurodegenerative disorder with motor and non-motor symptoms affecting approximately $1 \%$ of the population over 60 years, the prevalence of which increases with age ${ }^{1}$. As the disease progresses, there is an increasing rate and severity of motor features such as postural instability, falls or dysphagia ${ }^{2}$, as well as non-motor problems such as cognitive impairment, depression, anxiety, sleep disorders, apathy and psychosis ${ }^{3,4}$, which increase disability and reduce quality of life in the later disease stages 5 . As a consequence, patients in this late stage are typically highly dependent on caregivers for their activities of daily living.

Caring for a person with a neurodegenerative disease is a demanding process and may result in various negative physical, psychological and social consequences for caregivers ${ }^{6}$, which usually increase as the disease progresses and the patient gradually becomes more dependent on the caregiver ${ }^{7}$. With disease progression, patients' needs become more complex as they require increased assistance for daily activities (mobility, dressing, personal hygiene, eating, handling utensils) and medical requirements (administration of medication, attending medical appointments, therapy coordination) as well as communication ${ }^{2}$. Caregiver burden is a broad, multidimensional term describing the negative psychological state of caregivers induced by the demands of care for a person with chronic illness or disability ${ }^{6}$. Persistent burden may affect the caregiver's well-being and lead to physical (chronic illness) or psychological problems (emotional strain, depression, anxiety) ${ }^{8,9}$.

Late-stage parkinsonism patients and their caregivers constitute a complex group of participants that cannot easily be recruited in studies due to the severity of motor and non- 
motor problems, age, comorbidities as well as the fact that patients tend to withdraw from specialized health care in the late stage ${ }^{2}$. Only a few studies concentrated on the burden of caregivers of patients with advanced $\mathrm{PD}^{10-13}$ or atypical parkinsonism ${ }^{14-16}$ and their satisfaction with support ${ }^{17}$, which had relatively small samples, yielded inconsistent results regarding the contribution of motor, non-motor symptoms and caregiving factors to caregiver burden, and did not include an in-depth assessment. Therefore, aim of this study was to investigate caregivers' burden in late-stage parkinsonism patients and the factors associated with it. Identifying the factors that contribute to caregivers' burden could help develop interventions, in order to improve psychosocial outcomes for caregivers and consequently improve care of patients in these late stages.

\section{Patients and methods}

\section{Patients and caregivers}

Care of Late Stage Parkinsonism (CLaSP) study ${ }^{18}$ is a longitudinal, multicenter, prospective cohort study conducted from September 2014 to March 2019 assessing the needs and provision of care for patients with late-stage parkinsonism and their caregivers in six European countries. From the initial 588 patients and their primary informal caregivers from the crosssectional part of the CLaSP study, 506 (86.1\%) completed the Zarit Burden Interview (ZBI), while $82(13.9 \%)$ did not. The number of participants and response rate per site were as follows: 91 participants from the United Kingdom (UK) (87.5\% responders), 73 from France (97.3\%), 112 from Germany (75.2\%), 68 from the Netherlands (88.3\%), 86 from Portugal (93.5\%), 76 from Sweden (83.5\%). Responders were more often the spouse (57.7\% vs $18.3 \%$, $p<0.001)$ or child $(18.2 \%$ vs $7.3 \%, p=0.02)$ of the patient compared to non-responders. Additionally, responders received less often assistance from others ( $45.8 \%$ of responders had no assistance from others vs $24.1 \%$ of non-responders, $p=0.04)$. There was also a marginal difference regarding living with the patient ( $67 \%$ of responders vs $45.8 \%$ of non-responders, 
$p=0.06$ ), while there was no difference regarding employment state. Participants were recruited from neurology settings (hospital and office-based neurologists and psychiatrists, rehabilitation centers), nursing homes, palliative and primary care settings adapted to health care arrangements in each country. Inclusion criteria were late-stage parkinsonism i.e. disease duration of at least seven years and Hoehn and Yahr stage IV or V or significant disability (Schwab and England stage 50\% or less) in the "on"-state. Patients with potentially curable parkinsonism such as normal pressure hydrocephalus or drug-induced parkinsonism were excluded. Most of the patients (95\%) had idiopathic PD according to the UK PD society Brain Bank clinical diagnostic criteria ${ }^{19}$, but as distinction of different parkinsonian syndromes after longer disease duration is difficult and patients', patients with atypical parkinsonism (Multiple system atrophy-MSA, Progressive Supranuclear Palsy-PSP, Corticobasal Degeneration-CBD, Lewy body dementia-LBD) and vascular parkinsonism were also included if they had a disease duration of longer than 7 years. Patients were selected based on their health record and invited to participate by a letter from their clinician or they were referred by clinicians directly. The study was approved by the local ethics committees of all participating study sites and all participants gave their informed consent.

\section{Assessments}

Caregiver's burden was assessed by the $\mathrm{ZB}^{20}$, which is a 22 -item questionnaire assessing the physical, emotional, social and financial impact of the disease on the caregiver. Each item is answered on a 5-point scale from 0 (never) to 4 (nearly always). The ZBI ranges from 0-88 with higher scores indicating higher burden. The total score can be categorized as follows: $0-20$ (little or no burden), 21-40 (mild to moderate burden), 41-60 (moderate to severe burden), and $61-88$ (severe burden).

Patients' disease characteristics included age, gender and residential status; disease severity was assessed using the Unified PD rating scale (UPDRS) ${ }^{21}$, disease stage using Hoehn and Yahr 
scale ${ }^{22}$ and disability using the Schwab and England scale ${ }^{23}$. Mini-Mental-state examination (MMSE) ${ }^{24}$ was used to assess patients' general cognitive function. Non-motor symptoms were measured by the Non-Motor Symptom Scale (NMSS) ${ }^{25}$, which consists of 30 items grouped in nine domains: cardiovascular/falls, sleep/fatigue, mood/cognition, perceptual problems/hallucinations, attention/memory, gastrointestinal system, urinary symptoms, sexual function, miscellaneous. The score of each item is a multiple of frequency (1-4) and severity (0-3). Neuropsychiatric symptoms were assessed using the Neuropsychiatric inventory $(\mathrm{NPI}-12)^{26}$, a caregiver-based instrument assessing twelve neuropsychiatric symptoms (delusions, hallucinations, agitation/aggression, depression/dysphoria, anxiety, euphoria/elation, apathy/indifference, disinhibition, irritability/lability, aberrant motor behavior, nighttime behavior disorders, eating changes) in patients. The frequency and severity of symptoms within the last month are rated on a 3-point and 4-point Likert scale respectively, are multiplied and the subscores are then added to give the sum score. Patients' comorbidities were assessed by the Charlson Comorbidity Index $(\mathrm{CCI})^{27}$.

Informal caregiver information included their relationship to the patient, whether they lived with the patient, their current employment status and time providing care (hours per day and days per month assisting the patient and supervising the patient). Moreover, it included the proportion of care provided by the caregiver, whether additional assistance was provided and the frequency of contact with the patient if not living together. In addition, we assessed the caregivers' own health status using the EQ-5D-3L ${ }^{28}$. The EQ-5D-3L consists of two parts, one assessing the five dimensions of mobility, self-care, usual activities, pain/discomfort, anxiety/depression on three levels, and a visual analogue scale (EQ VAS), indicating the current own health status from 0 (worst imaginable health state) to 100 (best imaginable health state).

\section{Data analysis}


The statistical analysis was performed using the IBM SPSS 25.0. Descriptive statistics were used for sociodemographic and clinical variables. The normality of the variables was assessed using graphical methods and the Shapiro-Wilk test of normality. We included variables having $<10 \%$ missing data, in few variables with $>10 \%$ missing the number of cases $(\mathrm{N})$ is reported in parenthesis. To analyze the relationship between $\mathrm{ZBI}$ and other variables we performed correlation analyses using Pearson or Spearman rank correlations. Correlations were considered weak for $r$ values $\leq 0.29$, moderate from 0.3 to 0.59 and strong for values $\geq 0.6$. Statistical comparisons for non-normally distributed data were performed with the nonparametric Kruskal-Wallis or Mann-Whitney test. Comparisons were done using ANOVA for continuous variables and chi-squared test for categorical variables. Bonferroni and Scheffe correction were applied to adjust for multiple comparisons. A stepwise multiple regression analysis was performed considering the variables that showed significant correlations with ZBI (having $<10 \%$ missing data), after excluding collinearity between the variables. The level of significance was set at 0.05 .

\section{Results}

\section{Patient and caregiver characteristics}

The sociodemographic and clinical characteristics of patients and their caregivers are shown in Table 1 and 2. The majority of caregivers were the spouse or life partner of the patient (71.2\%), and $67 \%$ were living with the patient at home. Twenty three percent of caregivers were in employment. The severity of patients' motor and non-motor symptoms is seen in Table 1 and supplementary Table 1. Caregivers' characteristics can be seen in Table 2.

[Insert table 1 about here]

[Insert table 2 about here]

\section{Extend of caregiving}


On average, caregivers reported spending $6.7( \pm 6.6)$ hours per day and $23.1( \pm 10.6)$ days per month assisting with tasks of daily living (food preparation, housekeeping, shopping, transporting, administering medications, managing financial matters). Caregivers also reported spending $7.6( \pm 8.2)$ hours per day supervising the patient, in order to prevent dangerous events. Most caregivers (63.9\%) undertook at least $40 \%$ of care of the patient and half $(46.7 \%)$ more than $80 \%$ of the care (among all informal and professional care). Approximately half of the caregivers (54.21\%) reported that they had assistance from others, mainly another family member or friend. Additionally, almost half of the caregivers (49.2\%) reported assistance from professional services such as day or night care, cleaning service, food delivery, care-related transportation. Ninety percent of the caregivers $(90.7 \%)$, who did not live with the patient $(\mathrm{N}=132)$, reported spending $1.68( \pm 2.5)$ hours per day assisting and 1.82 $( \pm 4)$ hours per day supervising the patient. They visited the patient at least once a week (often several times a day) and $53.5 \%$ called them on the phone between once a week and several times a day. The majority of caregivers not living with the patient (70\%) were from 5 to more than 24 hours a week in contact with the patients (personally or on the phone). Out of the caregivers who were working (employed or self-employed, $\mathrm{N}=92$ ), 36 (39\%) were absent from their job at least one day in the last month and $6(6.5 \%)$ had to change or reduce their work during the last three months because of the patient's care.

Regarding care provision across the different countries caregivers spent statistically significant more hours assisting the patient in the UK $(8.23 \pm 7.14)$, Germany $(9.39 \pm 7.78)$ and France $(7.99 \pm 5.37)$ than in Sweden $(3.08 \pm 3.27)$ and the Netherlands $(3.02 \pm 4.41)(p<0.001)$. Caregivers in Germany spent statistically significant more hours supervising the patient (12.07 $\pm 9.17)$ than in the other countries $(p<0.001)$, while there was no statistical significant difference with regard to the years of caregiving across countries. Caregivers in France, Sweden and the Netherlands received statistical significant more professional care $(78 \%, 64 \%$, $56.5 \%$ of caregivers respectively) compared to the United Kingdom and Germany-(42.7\% and 
$34.6 \%$ respectively) $\left(\chi^{2}=38.380, p<0.001\right)$. Moreover, in all countries except for the Netherlands most caregivers were retired $\left(\chi^{2}=58.098, p<0.001\right)$. In the UK, Germany, Portugal and France caregivers were living more frequently with the patient $(85.4 \%, 81.1 \%, 77.8 \%$, $65.8 \%$ of caregivers respectively) compared to the Netherlands (32.3\%) and Sweden (55.6\%) $\left(\chi^{2}=62.627, p<0.001\right)$, where the patients' residential status was more often a nursing home than own home $(66.2 \%$ and $35.5 \%$ of patients in Netherlands and Sweden respectively were living in nursing homes versus $11 \%$ in the UK, $6.3 \%$ in Germany, $26.7 \%$ in Portugal and $27.4 \%$ in France $)\left(\chi^{2}=93.891, p<0.001\right)$.

\section{Caregiver's health status}

On the EQ-5D-3I, 58.7\% reported pain/discomfort, $45.5 \%$ anxiety/depression, $23.8 \%$ problems with mobility themselves, $24.3 \%$ problems with usual activities and $8 \%$ with self-care. Their mean health state score on the visual analogue scale was $73.1 \pm 19.5$ (Table 2).

\section{Burden of caregiving}

Five hundred and six caregivers completed the ZBI with a mean score $31.3 \pm 16$. There was no significant difference of ZBI score among the six European countries, except for a marginal higher caregiver burden in France compared to the Netherlands (mean ZBI score 35.11 vs $26.31, p=0.06$ ). For those still living with the patient at home (374 caregivers), the mean ZBI score was $32.47 \pm 15.76$. Amongst the most frequently endorsed statements at least some of the time were feeling that the patient depends on them (93.8\%), being afraid what the future holds for the patient $(90.3 \%)$, feeling burdened in caring for the patient $(81.8 \%)$ and not having enough time for themselves (80.5\%). More than seventy percent (77\%) felt stressed between caring for the patient and trying to meet other responsibilities for family or work, that their relative expects them to take care of them as the only one they could depend on (71\%) and that their social life and own health suffered because of their involvement with the patient's 
care $(71.4 \%$ and $67.1 \%$ respectively). At least some of the time many $(65.5 \%)$ also felt uncertain about what to do about the patient, that the patient asks for more help than they needed $(61.8 \%)$ and felt strained or angry when they were around the patient $(63.5 \%$ and $61.3 \%$ respectively). Approximately sixty percent (61.5\%) felt at least some of the time that they don't have as much privacy as they would like because of the patient, $61.2 \%$ wished at least sometimes that they should be doing more for the patient and $52.3 \%$ that they could leave the care of the patient to someone else. Moreover, the half (51\%) reported having at least sometimes felt embarrassed over the patient's behavior, $41.3 \%$ that the patient currently affects their relationships with other family members or friends in a negative way but only $27.5 \%$ ever felt uncomfortable about inviting friends at home because of the patient. Lastly, $47.9 \%$ felt at least sometimes they don't have enough money to take care of the patient. The caregivers' answers to the ZBI questions can be seen in supplementary Table 2 .

\section{Correlates of caregivers' burden}

The correlates of ZBI scores with patient and caregiver variables are shown in Tables 3 and 4. On patient-related variables, ZBI scores correlated most strongly with the total NPI score $(r=0.38, p<0.0001)$ followed by the total NMSS $(r=0.28, p<0.0001)$. Amongst the neuropsychiatric symptoms, the strongest correlations with caregiver burden were seen for agitation/aggression ( $r=0.23, p<0.0001)$, followed by apathy/indifference $(r=0.23, p<0.0001)$, disinhibition $(r=0.22, p<0.0001)$ and irritability/lability $(r=0.21, p<0.0001)$. With regard to NMSS domains, we found significant correlations of ZBI score with all domains except for sexual function, with the strongest correlations for attention/memory $(r=0.26, p<0.0001)$ and mood/cognition ( $r=0.22, p<0.0001)$. ZBI score correlated weakly with MMSE, Schwab and England score and with both UPDRS I (Mentation, Behavior and Mood) and II (Activities of Daily Living) ( $r=0.25, p<0.0001$ and $r=0.21, p<0.0001$ respectively) scores. However, there was no correlation with Hoehn and Yahr stage, UPDRS III (Motor Examination), UPDRS IV 
(dyskinesias and motor fluctuations) scores, disease duration, patient's age and the Charlson comorbidity index. Caring for a male patient was associated with higher ZBI ( $U=24057$, $p<0.0001$, with median ZBI scores 34 for male patients versus 26 for female), but there was no ZBI difference between the patient groups with different diagnoses (PD, atypical parkinsonism, vascular parkinsonism). Caregivers of patients living at home had increased burden compared to those living at nursing home $(U=20466.5$, median ZBI score 32 versus 25 , $p=0.003)$

Amongst caregiver-related variables, being the spouse as caregiver was associated with higher ZBI scores (median ZBI 34.5) as compared to being the child (median ZBI 26), other family member (median ZBI 18) or friend/neighbor (median ZBI 17) (Kruskal-Wallis test $=\chi^{2}{ }_{(3)}=24.64$, $p<0.0001, \mathrm{~N}=410)$. ZBI score also increased with hours per day $(\mathrm{r}=0.35, p<0.0001, \mathrm{~N}=367)$ and days per month assisting the patient $(r=0.21, p<0.0001, N=378)$ as well as hours per day supervising the patient $(r=0.37, p<0.0001, N=360)$. ZBI score correlated moderately with caregiver's EQ-5D-3L subscores for depression/anxiety $(r=0.37, p<0.0001)$ and pain/discomfort $(r=0.21, p<0.0001)$, but only weakly with the subscore for usual activities $(r=$ $0.18, p<0.0001)$. There was also a negative weak correlation with EQ-VAS scores $(r=-0.27$, $p<0.0001$ ) but no significant correlations with the EQ-5D-3L subscores for mobility or self-care.

[Insert table 3 about here]

[Insert table 4 about here]

\section{Determinants of caregivers' burden}

We performed a stepwise multiple regression analysis with ZBI score as dependent variable entering the variables that showed significant associations with ZBI score as independent variables. After excluding collinearity between the variables with both the variance inflation factor $(<3)$ and tolerance $(>0.2)$, we performed a multiple regression analysis within the subset 
of patients for which data with less than $10 \%$ missing data were available $(\mathrm{N}=445)$. The results showed that a model comprising NPI total score, patient's gender (male), residential status (living at home) and NMSS score could explain $23.3 \%$ of the total variance of the ZBI score $\left(R^{2}=0.233, p<0.0001\right)$. The predictor contributing the most to ZBI score was the NPI total score, which explained $14.7 \%$ of the variance.

[Insert table 5 about here]

\section{Discussion}

In this study, we found that caregivers of patients with late-stage parkinsonism experience considerable burden across physical, emotional and social aspects. High caregiver burden in PD, which increases as the disease progresses, has been reported previously ${ }^{8,29-31}$. The mean $\mathrm{ZBI}$ in this sample was higher than in patients with other physical disabilities and mild to moderate dementia ${ }^{32}$ and comparable or higher to that in caregivers of patients with more severe cognitive impairment ${ }^{32,33}$. Caregivers, who were in the great majority the spouse or life partner and living with the patient at home, reported spending on average 6.7 hours a day assisting the patient with activities and 7.6 hours supervising them. Only half received other informal or professional assistance for the patient's care. The percentage of caregivers receiving formal care assistance from professional services ( $49.2 \%$ overall, $42.7 \%$ in the UK) was higher than in a recent cohort of patients with advancing PD and their caregivers in the UK ${ }^{34,35}(26.16 \%)$, which might be attributed to the separate assessment of formal help with personal care and domestic care in the other study ${ }^{34}$. Moreover, caregiving hours were lower in the present study ( $6.7 \pm 6.6$ hours per day, $8.2 \pm 7.1$ in the UK) than in their study (median 16) ${ }^{34}$, which may be also attributed to discrepancies of definition (for example the study of Hand et al. ${ }^{34}$ included companionship activities among caregiver's tasks) as well as perception of caregiving from participants. Direct comparisons between these studies are therefore difficult but this suggests that our estimates of hours of informal caring and percentage of 
formal care may be due to differences in assessment. With regard to differences across countries, caregivers of the six countries had comparable ZBI scores. It is noteworthy that caregivers in Sweden and the Netherlands were living less often with the patient, spent fewer hours assisting the patient in daily living and received more frequently formal care assistance compared to the other countries. This may suggest differences in recruitment between participating centers but may also reflect different cultural perceptions of caregiving and attitude to nursing homes as well as the increasing development of modern nursing homes with person-centered care in these northern European countries ${ }^{36,37}$.

The majority of caregivers (approximately $80 \%$ ) felt stressed between caring for the patient and trying to meet other responsibilities, and felt that their social life or own health had suffered because of their involvement with the patient's care. Of note, almost half of the caregivers experienced anxiety and depression, a finding also reported in previous studies ${ }^{8,9}$, ${ }^{38-40}$. Caregiving for a person with a neurodegenerative disease is a chronic life stressor, causing feelings of isolation and grieve for the disruption of the caregivers' previously active lifestyle and family or relationship dynamic ${ }^{41}$. Additionally, because of the changes that caregivers' experience in their social or professional life, they often encounter feelings of frustration or uncertainty about the future ${ }^{42}$. In this study, these affective symptoms were associated with higher caregiver burden, as in many other previous studies ${ }^{40,43-46}$. They may be a consequence of the burden of caring or, alternatively, depression and anxiety may induce pessimism and burden of caregiving may be experienced as greater ${ }^{6}$. More than half of the caregivers reported pain or discomfort and to a lesser extent problems with mobility or usual activities themselves. It has previously been found that, caregivers report physical symptoms more frequently than non-caregiving individuals ${ }^{30,47}$. Apart from the increasing physical care of the patient in late stages of the disease (requiring feeding, lifting and transferring of the patient), advanced age of most caregivers, comorbidities as well as psychological distress may contribute to poorer physical health ${ }^{48,49}$. 
The clinical features of PD most closely associated with increased caregiver burden were the patients' non-motor symptoms with only weak relationship with motor problems. This however needs to be considered in the context of a study where all patients were in the late stages of the disease. Nevertheless, non-motor symptoms have also previously been reported to make a greater contribution to caregiver burden with only weak associations with motor scores or complications in early and middle PD stages ${ }^{50-52}$. Particularly, the importance of neuropsychiatric symptoms and their contribution to caregiver burden has also been reported previously ${ }^{13,40,50,53-55}$. Amongst the neuropsychiatric symptoms, the strongest associations were found with apathy, agitation/aggression, disinhibition and irritability of the patient in our study. Apathy, which includes loss of interests, motivation and empathy, can affect the relationship with the caregiver by reducing the emotional feedback received by the caregiver ${ }^{6}$ or being misinterpreted as an act of non-engagement ${ }^{41}$. This can cause negative feelings to the caregiver and strain the spousal dynamic ${ }^{41}$. Disinhibition such as impulsive behavior or hypersexuality as a result of the dopaminergic treatment is another neuropsychiatric feature that has been linked to caregiver burden ${ }^{56}$. Bruno et al. ${ }^{56}$ reported high rates of both physical and sexual aggression directed against the caregiver in advanced PD. The cause of aggression is likely multifactorial including cognitive decline, depression, anxiety, loss of independence and medication effects. These behaviors apart from the psychological strain, can make the caregiver (spouse or life partner) question the marital relationship or partnership ${ }^{41}$. We also found a correlation of caregiver burden with the attention/memory and mood/cognition domains of the NMSS. Almost $40 \%$ of the patients had a concurrent diagnosis of dementia based on clinical diagnostic criteria ${ }^{57}$, which is a common complication of advancing $\mathrm{PD}^{58}$. A number of studies $50,54,59,60$ have stressed the contribution of patient's cognitive impairment to caregiver's burden. The executive dysfunction of patients can lead to caregivers taking on complex cognitive tasks (such as finances or planning), with increased time demands and distress ${ }^{41}$. Sleep and nighttime behavior disorders are also a common non-motor problem in 
$P D^{61}$, reported for almost half of the patients in the present study (suppl. Table 1). Caregiver's sleep quality is frequently disturbed due to insomnia, sleep fragmentation, hallucinations or disturbed motor activity such as REM behavior disorder and Restless Leg Syndrome of patients ${ }^{61}$, requiring additional night time care ${ }^{41}$. In keeping with previous reports that PD patients' sleep quality affects caregivers' mood, quality of life and burden ${ }^{39,62}$, we found significant correlations of patients' sleep disturbance as assessed on the NMSS with caregiver burden.

Caring for a male patient was associated with higher burden among caregivers in the present study. This may be due to greater physical demands of care for a male patient but possibly also to the disinhibition such as impulsive behavior or hypersexuality, which is more common in male patients ${ }^{58,63}$. As expected, burden was higher among caregivers of patients still living at home as opposed to caregivers of patients living in a nursing home, as in those living at home the caregiver is the main assistance in daily living. Increased caregiver strain is a strong predictor of patient's institutionalization ${ }^{64,65}$, and the reduced burden of caregivers of PD patients living at nursing homes may reflect the alleviation of caregiver's "burnout" ${ }^{64}$. This is supported by the significant lower number of hours of caregiving reported by caregivers of those living in nursing homes in our study. We found that the hours assisting and supervising the patient were important contributors to caregiver burden. While studies reported inconsistent findings regarding hours of caregiving as predictor for burden ranging from no correlation ${ }^{45,52}$ to a weak or moderate correlation ${ }^{30,46,66}$, the meta-analysis of Lau and $\mathrm{Au}^{55}$ showed that caregiver burden was determined by the intensity of caregiving, measured by the number of caregiving hours and years. In the present study there was no difference of ZBI scores between the patient groups with different diagnoses (PD, atypical parkinsonism, vascular parkinsonism), but the number of patients with other parkinsonian disorders was small. 
As only $23.3 \%$ of the total variance of the caregivers' burden was explained from the patient and caregiver characteristics assessed i.e. NPI total score, patient's gender (male), residential status (living at home) and NMSS score, it is likely that other factors - personal, cultural or social - contribute to caregivers' burden such as caregivers' comorbidities and social support, which should be explored in separate studies. Indeed, caregiver burden is a general term that reflects the impact of caregiving on persons from different backgrounds and with different levels of resilience and resources ${ }^{6}$. Goldsworthy and Knowles in their stress-appraisal model ${ }^{67}$ acknowledge that apart from disease factors, which act as primary stressors for the caregiver, individuals (caregivers) respond differently to the patient's disease undergoing two levels of stress-appraisal i.e. cognitive, emotional, behavioral processes, which help them cope with the stressful event. Perceived positive aspects of caregiving such as the intimacy of the caregiving relationship ${ }^{67}$, the feeling of being useful and able to manage difficult situations ${ }^{46,60}$, the caregiver's personality traits ${ }^{68}$, self-esteem ${ }^{67}$ and perceptions in life seem also to be important predictors of caregiver burden. We did not assess these factors which are likely to contribute to caregiver strain in addition to the factors that we assessed.

\section{Strengths and Limitations}

This is the largest and most comprehensive study of caregiver burden in late-stage PD to our knowledge, providing new information not only on the amount of care provided by informal caregivers, but also on the factors that contribute the most to the caregiver burden. Strengths of the study include the large patient and caregiver sample, the multicenter design protocol and the use of a wide range of standardized validated measures for the assessment of PD and caregivers. One limitation of this study is that caregiver burden was assessed using the ZBI, which although being an established validated caregiver burden measure, is not a PD-specific questionnaire such as the Parkinson's Disease Caregiver Burden questionnaire (PDCB) ${ }^{69,70}$ or an instrument relevant for patients with advanced parkinsonism such as the Parkinsonism 
Carers Quality of Life (PQoL Carer) ${ }^{71}$. It cannot be excluded that some parkinsonism-specific issues were missed through the use of the ZBI, which may account for the discrepancy with other studies ${ }^{10,48}$. Another limitation of the study is that only age and gender of the patient and not age and gender of the caregiver were included in the analysis. However, we used patient age and gender as proxies. Furthermore, some caregivers' variables had missing data $(<20 \%)$, which is inevitable in such large participants samples. However we feel that this did not affect the overall results as these variables were not entered in the multiple regression model and replaced from equivalent ones (e.g. cognition domain of the NMSS instead of MMSE). Particularly, MMSE could only be completed by 315 patients and we cannot exclude that those patients who were cognitively worse did not complete the test. Moreover, there were differences between ZBI responders and non-responders in terms of relationship with the patient and assistance received from others.

This study gives insights into the needs of patients with an advanced neurodegenerative disease and their caregivers, which may inform interventions in clinical practice. Non-motor symptoms should be addressed with focus on those being the most bothersome for both patient and caregiver. Of particular importance are the identification and treatment of neuropsychiatric symptoms, to improve patient but also caregiver burden. Pharmacological and non-pharmacological management of neuropsychiatric symptoms ${ }^{72}$ could be implemented by caregivers' interventions, including counseling, demonstration of deescalation techniques and psychoeducation regarding neurobehavioral changes ${ }^{56}$. The high caregiver burden in the late stages, especially for the spouse or life partner, identifies them as an important group to consider for interventions in their own right. For example, cognitive behavioral therapy for the caregiver focusing on pleasant activity scheduling, relaxation techniques, sleep improvement, identifying and avoiding negative thoughts have been reported to have positive effects on their burden ${ }^{73}$. Goldsworthy and Knowles proposed coping mechanisms such as social support, ameliorating quality of patient-caregiver 
relationship or breaks in caregiving ${ }^{67}$. The aim of these interventions is to help caregivers adapt their perception and behavior to the changes in their life caused by the patient's disease.

Our findings have implications in organizing targeted management of symptoms in patients with late-stage parkinsonism, in order to improve patients' and caregivers' quality of life. The close links between patient's disease characteristics and caregiver burden suggest a complex physician-patient-caregiver system, so that interventions should involve both patient and caregiver. Patients in the late stages are also increasingly dependent on health systems and caregivers require special education on the associated features and treatment to support the patient and navigate the healthcare and social system. From a societal perspective, effective caregiving not only contributes to the management of the disease-related problems but has also financial benefits by substantially reducing health care costs such as hospital admissions, polypharmacy and delaying institutionalization of the patient ${ }^{74}$. Patients with late-stage parkinsonism have special needs requiring the engagement of multiple specialties (especially neurology, psychiatry, internal medicine, geriatrics) and palliative care services (hospice and nursing service, advanced care such as feeding and airway management, end-of-life planning $)^{5}$. Thus, it is essential to have an interdisciplinary network of care to address all their medical issues in a holistic, individual-tailored based concept ${ }^{75}$ providing sufficient support for them and their caregivers.

Acknowledgement: We thank all the patients and carers who participated in this study, and Kareem Khan, Gergely Bartl, Christian Schmotz, Carmen Richinger, Fabrice Blard, Thomas Boraud, Morgane Gaboreau, Brice Laurens, Umberto Spampinato, Sylvain Vergnet, Jonathan Timpka for data collection. 


\section{Funding}

The study was funded by the European Commission (Joint Programme - Neurodegenerative Disease Research "European research projects for the evaluation of health care policies, strategies and interventions for Neurodegenerative Diseases") through national funding bodies in all six countries (Economic and Social Research Council ES/L009250/1; BMBF, Marburg, Germany 01ED1403A, Munich, Germany 01ED1403B, Bordeaux, France: ANR-13JPHC-0001-07, Lisbon, Portugal: HC/ 0002/2012, Lund, Sweden: HC-559-002, Nijmegen, Holland, 733051003). AS was supported by the National Institute for Health Research UCL/UCLH Biomedical Research Centre. AH was supported by co-funding of Groenhuysen organization and Stichting Beroepsopleiding Huisartsen.

\section{Conflicts of interest}

The authors declare that there is no conflict of interest.

\section{Appendix}

Members of the CLaSP Consortium: Margherita Fabbri, François Tison, Alexandra FoubertSamier, Joy Read, Marjan Meinders, Raymond Koopmans, Carmen Richinger, Kristina Rosqvist, Michael Wittenberg, Petra Neuser

\section{References}

1. Tysnes $\mathrm{OB}$, Storstein A. Epidemiology of Parkinson's disease. Journal of neural transmission. 2017;124(8):901-905.

2. Coelho M, Ferreira JJ. Late-stage Parkinson disease. Nature reviews Neurology. 2012;8(8):435-442.

3. Schapira AHV, Chaudhuri KR, Jenner P. Non-motor features of Parkinson disease. Nature reviews Neuroscience. 2017;18(7):435-450.

4. Barone $\mathrm{P}$, Antonini A, Colosimo C, et al. The PRIAMO study: A multicenter assessment of nonmotor symptoms and their impact on quality of life in Parkinson's disease. Movement disorders : official journal of the Movement Disorder Society. 2009;24(11):1641-1649.

5. Klietz M, Tulke A, Müschen LH, et al. Impaired Quality of Life and Need for Palliative Care in a German Cohort of Advanced Parkinson's Disease Patients. Frontiers in neurology. 2018;9:120. 
6. Mosley PE, Moodie R, Dissanayaka N. Caregiver Burden in Parkinson Disease: A Critical Review of Recent Literature. Journal of geriatric psychiatry and neurology. 2017;30(5):235252.

7. Greenwell K, Gray WK, van Wersch A, et al. Predictors of the psychosocial impact of being a carer of people living with Parkinson's disease: a systematic review. Parkinsonism \& related disorders. 2015;21(1):1-11.

8. Carod-Artal FJ, Mesquita HM, Ziomkowski S, et al. Burden and health-related quality of life among caregivers of Brazilian Parkinson's disease patients. Parkinsonism \& related disorders. 2013;19(11):943-948.

9. Meara J, Mitchelmore E, Hobson P. Use of the GDS-15 geriatric depression scale as a screening instrument for depressive symptomatology in patients with Parkinson's disease and their carers in the community. Age and ageing. 1999;28(1):35-38.

10. Klietz M, Schnur T, Drexel S, et al. Association of Motor and Cognitive Symptoms with Health-Related Quality of Life and Caregiver Burden in a German Cohort of Advanced Parkinson's Disease Patients. Parkinson's disease. 2020;2020:5184084.

11. Tessitore A, Marano P, Modugno N, et al. Caregiver burden and its related factors in advanced Parkinson's disease: data from the PREDICT study. Journal of neurology. 2018;265(5):1124-1137.

12. Schmotz C, Richinger C, Lorenzl S. High Burden and Depression Among Late-Stage Idiopathic Parkinson Disease and Progressive Supranuclear Palsy Caregivers. Journal of geriatric psychiatry and neurology. 2017;30(5):267-272.

13. Oh YS, Lee JE, Lee PH, et al. Neuropsychiatric symptoms in Parkinson's disease dementia are associated with increased caregiver burden. Journal of movement disorders. 2015;8(1):26-32.

14. Armstrong N, Schupf N, Grafman J, et al. Caregiver burden in frontotemporal degeneration and corticobasal syndrome. Dementia and geriatric cognitive disorders. 2013;36(5-6):310-318.

15. Miyashita M, Narita $Y$, Sakamoto A, et al. Care burden and depression in caregivers caring for patients with intractable neurological diseases at home in Japan. Journal of the neurological sciences. 2009;276(1-2):148-152.

16. Uttl B, Santacruz P, Litvan I, et al. Caregiving in progressive supranuclear palsy. Neurology. 1998;51(5):1303-1309.

17. Rosqvist K, Hagell $P$, Iwarsson $S$, et al. Satisfaction with Care in Late Stage Parkinson's Disease. Parkinson's disease. 2019;2019:2593547.

18. Balzer-Geldsetzer M, Ferreira J, Odin P, et al. Study protocol: Care of Late-Stage Parkinsonism (CLaSP): a longitudinal cohort study. BMC neurology. 2018;18(1):185.

19. Hughes AJ, Daniel SE, Kilford L, et al. Accuracy of clinical diagnosis of idiopathic Parkinson's disease: a clinico-pathological study of 100 cases. Journal of neurology, neurosurgery, and psychiatry. 1992;55(3):181-184.

20. Zarit SH, Reever KE, Bach-Peterson J. Relatives of the impaired elderly: correlates of feelings of burden. The Gerontologist. 1980;20(6):649-655.

21. Fahn S ERaMotuDC. Recent Developments in Parkinson's Disease. In: Fahn S, Marsden CD, Calne DB, Goldstein M, eds. 1987; Vol 2.(Macmillan Health Care Information 1987, Florham Park, NJ.):pp 153-163, 293-304.

22. Hoehn MM, Yahr MD. Parkinsonism: onset, progression and mortality. Neurology. 1967;17(5):427-442.

23. Schwab RSE, A.C. Projection technique for evaluating surgery in Parkinson's disease. In Gillingham FJD, M.C, (Ed). 3rd Symposium on Parkinson's Disease. Livingstone, Edinburgh 1969:152-157.

24. Folstein MF, Folstein SE, McHugh PR. "Mini-mental state". A practical method for grading the cognitive state of patients for the clinician. J Psychiatr Res. 1975;12(3):189-198. 
25. Chaudhuri KR, Martinez-Martin P, Brown RG, et al. The metric properties of a novel non-motor symptoms scale for Parkinson's disease: Results from an international pilot study. Movement disorders : official journal of the Movement Disorder Society. 2007;22(13):19011911.

26. Cummings JL, Mega M, Gray K, et al. The Neuropsychiatric Inventory: comprehensive assessment of psychopathology in dementia. Neurology. 1994;44(12):2308-2314.

27. Charlson $M E$, Pompei $\mathrm{P}$, Ales $\mathrm{KL}$, et al. A new method of classifying prognostic comorbidity in longitudinal studies: development and validation. Journal of chronic diseases. 1987; 40(5):373-383.

28. EuroQol. EuroQol--a new facility for the measurement of health-related quality of life. Health policy (Amsterdam, Netherlands). 1990;16(3):199-208.

29. Carter JH, Stewart BJ, Archbold PG, et al. Living with a person who has Parkinson's disease: the spouse's perspective by stage of disease. Parkinson's Study Group. Movement disorders : official journal of the Movement Disorder Society. 1998;13(1):20-28.

30. Martinez-Martin P, Forjaz MJ, Frades-Payo B, et al. Caregiver burden in Parkinson's disease. Movement disorders : official journal of the Movement Disorder Society. 2007;22(7):924-931; quiz 1060.

31. Razali R, Ahmad F, Rahman FN, et al. Burden of care among caregivers of patients with Parkinson disease: a cross-sectional study. Clinical neurology and neurosurgery. 2011;113(8):639-643.

32. Rodriguez-Gonzalez AM, Rodriguez-Miguez E. A meta-analysis of the association between caregiver burden and the dependent's illness. Journal of women \& aging. 2020;32(2):220-235.

33. Igarashi A, Fukuda A, Teng L, et al. Family caregiving in dementia and its impact on quality of life and economic burden in Japan-web based survey. Journal of market access \& health policy. 2020;8(1):1720068.

34. Hand A, Oates LL, Gray WK, et al. The role and profile of the informal carer in meeting the needs of people with advancing Parkinson's disease. Aging \& mental health. 2019;23(3):337-344.

35. Hand A, Oates LL, Gray WK, et al. Understanding the Care Needs and Profile of People Living at Home With Moderate to Advanced Stage Parkinson Disease. Journal of geriatric psychiatry and neurology. 2018;31(5):237-247.

36. Edebalk PG. Ways of Funding and Organising Elderly Care in Sweden. Population Ageing - A Threat to the Welfare State? . Berlin Heidelberg: Springer-Verlag 2010:65-80.

37. Smits $\mathrm{CH}$, van den Beld HK, Aartsen MJ, et al. Aging in the Netherlands: state of the art and science. The Gerontologist. 2014;54(3):335-343.

38. Happe $S$, Berger K. The association between caregiver burden and sleep disturbances in partners of patients with Parkinson's disease. Age and ageing. 2002;31(5):349-354.

39. Pal PK, Thennarasu K, Fleming J, et al. Nocturnal sleep disturbances and daytime dysfunction in patients with Parkinson's disease and in their caregivers. Parkinsonism \& related disorders. 2004;10(3):157-168.

40. Schrag A, Hovris A, Morley D, et al. Caregiver-burden in parkinson's disease is closely associated with psychiatric symptoms, falls, and disability. Parkinsonism \& related disorders. 2006;12(1):35-41.

41. Hiseman JP, Fackrell R. Caregiver Burden and the Nonmotor Symptoms of Parkinson's Disease. International review of neurobiology. 2017;133:479-497.

42. Williamson C, Simpson J, Murray CD. Caregivers' experiences of caring for a husband with Parkinson's disease and psychotic symptoms. Social science \& medicine (1982). 2008;67(4):583-589. 
43. Macchi ZA, Koljack CE, Miyasaki JM, et al. Patient and caregiver characteristics associated with caregiver burden in Parkinson's disease: a palliative care approach. Annals of palliative medicine. 2020;9(Suppl 1):S24-s33.

44. Caap-Ahlgren M, Dehlin O. Factors of importance to the caregiver burden experienced by family caregivers of Parkinson's disease patients. Aging clinical and experimental research. 2002;14(5):371-377.

45. Martinez-Martin P, Arroyo S, Rojo-Abuin JM, et al. Burden, perceived health status, and mood among caregivers of Parkinson's disease patients. Movement disorders : official journal of the Movement Disorder Society. 2008;23(12):1673-1680.

46. Zhong $M$, Peppard $R$, Velakoulis $D$, et al. The relationship between specific cognitive defects and burden of care in Parkinson's disease. International psychogeriatrics. 2016;28(2):275-281.

47. Miyashita $M$, Narita $Y$, Sakamoto $A$, et al. Health-related quality of life among community-dwelling patients with intractable neurological diseases and their caregivers in Japan. Psychiatry and clinical neurosciences. 2011;65(1):30-38.

48. McLaughlin D, Hasson F, Kernohan WG, et al. Living and coping with Parkinson's disease: perceptions of informal carers. Palliative medicine. 2011;25(2):177-182.

49. Pinquart $\mathrm{M}$, Sorensen $\mathrm{S}$. Correlates of physical health of informal caregivers: a metaanalysis. The journals of gerontology Series B, Psychological sciences and social sciences. 2007;62(2):P126-137.

50. Aarsland D, Larsen JP, Karlsen K, et al. Mental symptoms in Parkinson's disease are important contributors to caregiver distress. International journal of geriatric psychiatry. 1999;14(10):866-874.

51. Carter JH, Stewart BJ, Lyons KS, et al. Do motor and nonmotor symptoms in PD patients predict caregiver strain and depression? Movement disorders : official journal of the Movement Disorder Society. 2008;23(9):1211-1216.

52. Shin $\mathrm{H}$, Lee JY, Youn J, et al. Factors contributing to spousal and offspring caregiver burden in Parkinson's disease. European neurology. 2012;67(5):292-296.

53. D'Amelio M, Terruso V, Palmeri B, et al. Predictors of caregiver burden in partners of patients with Parkinson's disease. Neurological sciences : official journal of the Italian Neurological Society and of the Italian Society of Clinical Neurophysiology. 2009;30(2):171174.

54. Aarsland D, Bronnick K, Ehrt U, et al. Neuropsychiatric symptoms in patients with Parkinson's disease and dementia: frequency, profile and associated care giver stress. Journal of neurology, neurosurgery, and psychiatry. 2007;78(1):36-42.

55. Lau K-MA, A. . Correlates of Informal Caregiver Distress in Parkinson's Disease: A Meta-Analysis. . Clinical Gerontologist. 2011;34(2):117-131.

56. Bruno V, Mancini D, Ghoche R, et al. High prevalence of physical and sexual aggression to caregivers in advanced Parkinson's disease. Experience in the Palliative Care Program. Parkinsonism \& related disorders. 2016;24:141-142.

57. Emre M, Aarsland D, Brown R, et al. Clinical diagnostic criteria for dementia associated with Parkinson's disease. Movement disorders : official journal of the Movement Disorder Society. 2007;22(12):1689-1707; quiz 1837.

58. Cooney JW, Stacy M. Neuropsychiatric Issues in Parkinson's Disease. Current neurology and neuroscience reports. 2016;16(5):49.

59. Leroi I, McDonald K, Pantula $\mathrm{H}$, et al. Cognitive impairment in Parkinson disease: impact on quality of life, disability, and caregiver burden. Journal of geriatric psychiatry and neurology. 2012;25(4):208-214.

60. Thommessen B, Aarsland D, Braekhus A, et al. The psychosocial burden on spouses of the elderly with stroke, dementia and Parkinson's disease. International journal of geriatric psychiatry. 2002;17(1):78-84. 
61. Menza $\mathrm{M}$, Dobkin RD, Marin H, et al. Sleep disturbances in Parkinson's disease. Movement disorders : official journal of the Movement Disorder Society. 2010;25 Suppl 1(Suppl 1):S117-122.

62. Bartolomei L, Pastore A, Meligrana L, et al. Relevance of sleep quality on caregiver burden in Parkinson's disease. Neurological sciences : official journal of the Italian Neurological Society and of the Italian Society of Clinical Neurophysiology. 2018;39(5):835839.

63. Aarsland D, Kramberger MG. Neuropsychiatric Symptoms in Parkinson's Disease. Journal of Parkinson's disease. 2015;5(3):659-667.

64. Abendroth $M$, Lutz BJ, Young ME. Family caregivers' decision process to institutionalize persons with Parkinson's disease: a grounded theory study. International journal of nursing studies. 2012;49(4):445-454.

65. McLennon SM, Habermann B, Davis LL. Deciding to institutionalize: why do family members cease caregiving at home? The Journal of neuroscience nursing : journal of the American Association of Neuroscience Nurses. 2010;42(2):95-103.

66. Peters $M$, Fitzpatrick $R$, Doll $H$, et al. Does self-reported well-being of patients with Parkinson's disease influence caregiver strain and quality of life? Parkinsonism \& related disorders. 2011;17(5):348-352.

67. Goldsworthy B, Knowles S. Caregiving for Parkinson's disease patients: an exploration of a stress-appraisal model for quality of life and burden. The journals of gerontology Series B, Psychological sciences and social sciences. 2008;63(6):P372-376.

68. Lyons KS, Stewart BJ, Archbold PG, et al. Optimism, pessimism, mutuality, and gender: predicting 10-year role strain in Parkinson's disease spouses. The Gerontologist. 2009;49(3):378-387.

69. Zhong M, Evans A, Peppard R, et al. Validity and reliability of the PDCB: a tool for the assessment of caregiver burden in Parkinson's disease. International psychogeriatrics. 2013;25(9):1437-1441.

70. Klietz M, Rippena L, Lange F, et al. Validating the Parkinson's disease caregiver burden questionnaire (PDCB) in German caregivers of advanced Parkinson's disease patients. International psychogeriatrics. 2019;31(12):1791-1800.

71. Pillas M, Selai C, Quinn NP, et al. Development and validation of a carers quality-oflife questionnaire for parkinsonism (PQoL Carers). Quality of life research : an international journal of quality of life aspects of treatment, care and rehabilitation. 2016;25(1):81-88.

72. Seppi K, Ray Chaudhuri K, Coelho M, et al. Update on treatments for nonmotor symptoms of Parkinson's disease-an evidence-based medicine review. Movement disorders : official journal of the Movement Disorder Society. 2019;34(2):180-198.

73. Secker DL, Brown RG. Cognitive behavioural therapy (CBT) for carers of patients with Parkinson's disease: a preliminary randomised controlled trial. Journal of neurology, neurosurgery, and psychiatry. 2005;76(4):491-497.

74. Oliva-Moreno J, Trapero-Bertran M, Pena-Longobardo LM, et al. The Valuation of Informal Care in Cost-of-Illness Studies: A Systematic Review. PharmacoEconomics. 2017;35(3):331-345.

75. Titova N, Chaudhuri KR. Non-motor Parkinson disease: new concepts and personalised management. The Medical journal of Australia. 2018;208(9):404-409. 